\title{
The Application of Real-time Imaging Detection Technology in the Detection of Aluminum Casting Parts
}

\author{
Yu Yang ${ }^{1, a}$, Dong Chen ${ }^{2}$ \\ ${ }^{1}$ Changchun University of Science and Technology,Changchun,Jilin province,China \\ ${ }^{2}$ Guangdong Ningyuan Science Park Development Company Limited \\ ayangyu1983@sina.com
}

\begin{abstract}
Key words:X-ray; real-time imaging; detection technology
Abstract.Modern X-ray real-time imaging detection technology is based on image intensifier ray imaging technology, using CCD camera and image collection and processing system, which observes produced image with transillumination. This paper focuses on batch detection requirements on product parts of a specific business, adopting X-ray real-time imaging detection technology to detect stomata, inclusion and porosity in aluminum casting. The results indicate that the resolution of detection is $32 \mathrm{PL} / \mathrm{cm}$, and the sensitivity is $1.8 \%$.
\end{abstract}

\section{Introduction}

With the improvement of image intensifier, CCD imaging and image collection and processing technology, X-ray real-time imaging detection technology is more and more applied in production $^{[1-6]}$. The factors that affect imaging quality of real-time imaging detection are resolution and sensitivity of the system. In this research on X-ray non-destructive testing equipment, real-time imaging detection technology analyses the factors affecting imaging quality and offers methods to improve image quality and detection precision.

\section{Theory of Ray Detection}

The wavelength range of X-ray is 0.01-100 A.The wavelength of those for medical applications is $0.01-1 \AA$ and for medical diagnosis is $0.1-1 \AA$. The shortest wavelength of extreme soft X-ray used in photographing of soft tissue and treating epidermis is $2.5-0.62 \AA$. The shortest wavelength of soft $X$-ray used in perspective and photographing is $0.62-0.12 \AA$. The shortest wavelength of hard X-ray used in treating deeper issue is 0.12-0.05 $\AA$. The shortest wavelength range of extra hard X-ray used in treating deep tissue and industrial flaw detection is below $0.05 \AA$.

The penetrability of X-ray used in industrial flaw detection is not only related with the energy size of X-ray but also the structure and atomic property of the penetrated material. Larger energy of photon means a shorter wavelength of the X-ray produced and a lower penetration rate of X-ray. Higher atomic number means larger density, a larger quantity of X-ray absorbed and a lower penetration rate of X-ray. On the contrary, lower atomic number means lower density, a smaller quantity of X-ray absorbed and a higher penetration rate of X-ray.

When a ray of uniform intensity transilluminates objects, it will change the damping of objects to rays and make the transmission intensity of different parts distinct, if there are defects in local objects and differences in structures. To detect the transmission intensity of rays can be used to judge inner defects of objects. The main factors that affect detection precision in ray detection are contrast ratio of rays and boundary quality of ray image, in which image noise and X-ray focus size play a decisive role.

A. Contrast ratio of rays

Due to absorption and scattering of X-ray by illuminated objects, the rays through objects are uneven distributed, which is the contrast ratio of rays. According to the attenuation law of X-ray in materials, we can get the following formula.

$$
I=I_{0} e^{-\mu T}
$$


$I_{0}$ is the intensity of incident ray; $I$ is the intensity of transmission; $\mu$ is the linear attenuation coefficient; $T$ is the thickness of the absorber.

When rays penetrate different objects, the intensity of transmission are distinct because of different attenuation coefficient and thickness. The difference of intensity is the contrast ratio of rays, written as $K$.

$$
K=\frac{I_{2}}{I_{1}}=\frac{I_{0} e^{-\mu_{2} T_{2}}}{I_{0} e^{-\mu_{1} T_{1}}}=e^{\eta_{1} T_{1}-\mu_{2} T_{2}}
$$

The formula (2) is the basic principle relation of rays detection, which provides the relationship between objects of rays detection and contrast ratio. From the relation, we can find out that the detection ability of rays on defects is related to the measurement of defects in transillumination direction and the linear attenuation coefficient. As long as there is a value, and defects produce contrast ratio, it will be detected by rays. In the actual system, the obvious flicker noise of intensifier image results in the decline of image ratio.

$B$. Quality of the image boundary

The basic factor of boundary quality is the penumbra effect of X-ray focus size in rays detection.

Figure1 is distribution of ray blackness. When X-ray transilluminates the boundary of a sharp vertical metal object, blackness distribution curve of one step type will be obtained ideally. That is to say, as it transfers from one thickness to another, the corresponding blackness will transfer to another one in the form of step type. Because the focus of X-ray is not a ideal point light but with a certain measurement, the actual blackness distribution is not step type. It is a curve and a slowly changing area between two blackness, in which one blackness transfer to another. Flicker noise makes the changes become irregular and up-and-down rather than monotonous and uniform.

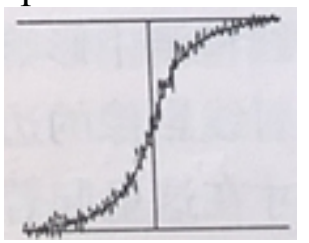

Figure1 Distribution of ray blackness

In the system of X-ray real-time imaging detection, we can improve the above influence factors in two ways.

1)Computer image processing technology can be used to improve image ratio and decrease the irregular changes of blackness curve by multi-frame superposing flick noise.

2)Adopting small focus X-ray tube to decrease the transition zone length of blackness distribution curve caused by decreasing penumbra field. Increase the slope of blackness distribution curve so as to make it close to step changes as much as possible.

\section{Image motion compensation}

As it is a real-time detection conducted in the assembly line, there will be a slow relative motion between the tested work-pieces and the testing system during testing. If the picture processing part at the final stage is not handled, the pictures will become fuzzy to see clearly whether there are defects in the work pieces. Consequently, image motion compensation will be utilized to restore the images.

In image restoration, we usually suppose transmission system as linear system. Original image $\mathrm{f}(\mathrm{x}, \mathrm{y})$ gets image $\mathrm{g}(\mathrm{x}, \mathrm{y}), \mathrm{n}(\mathrm{x}, \mathrm{y})$ as noise model by system $\mathrm{h}(\mathrm{x}, \mathrm{y})$. It is shown in Figure2.

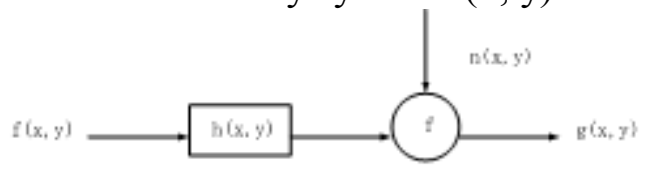

Figure2 Image degradation model

According to the degradation model, digital image restoration can be seen as a process to evaluate original image $f(x, y)$, based on degradation image $g(x, y)$, priori knowledge about $h(x, y)$ 
and system character about noise $\mathrm{n}(\mathrm{x}, \mathrm{y})$. And the evaluated image should be as close to the original one as possible. Degradation model is presented as:

$$
g(x, y)=f(x, y) \cdot h(x, y)+n(x, y)
$$

\section{Experiment of Ray Detection}

Figure 3 is detected workiece.

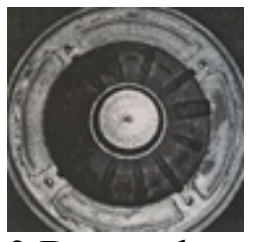

Figure3 Detected workiece

The requirements of detection are that less than three bubbles, shrinkage cavity and inclusion whose size and depth are smaller than $0.5 \mathrm{~mm}$ and $0.4 \mathrm{~mm}$ are allowed. Shrinkage porosity is not permitted. The defect of $0.4 \mathrm{~mm}$ must be recognized in detection.

$\mathrm{X}$-ray real-time imaging detection system employs image intensifier real-time imaging system. Its equipment is shown as followed.The composition of the equipment is shown in Figure4.

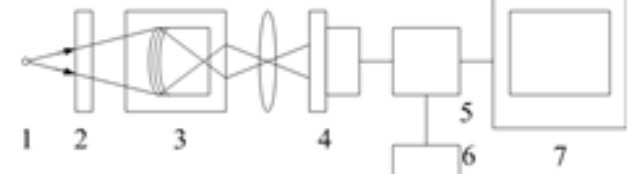

Figure4 The X-ray real-time imaging detection system

$1 \mathrm{X}$-ray tube 2 Detected workpiece 3Image intensifier 4.CCD camera 5 Computer 6Image processing software 7 Display

Parameters of workpiece:

1)X-ray tube: COMET MRX-225/22; Voltage $0 \sim 225 \mathrm{kV}$; Electricity $0 \sim 30 \mathrm{~mA}$ adjusting continuance; Big focus $1.5 \times 1.5 \mathrm{~mm}$; Small focus $0.4 \times 0.4 \mathrm{~mm}$.

2)Image intensifier: Toshiba E5830SD; Effective viewing field 215/160/120mm; resolution ratio $52 / 58 / 68 \mathrm{Lp} / \mathrm{cm}$.

3)CCD camera: Effective pixels $1024 \times 1024$; High speed interface camera link; Frame rate $30 \mathrm{fps}$.

Experiment conditions:

1)voltage of X-ray tube: $51 \mathrm{kV}$, electricity $15 \mathrm{~mA}$, small focus.

2)image intensifier $160 \mathrm{~nm}$ viewing field, resolution ratio $58 \mathrm{Lp} / \mathrm{cm}$.

The detection of bubbles, shrinkage cavity and inclusion of hemispherical aluminum casting achieves good results.

\section{Results of Experiment}

Figure5 is photo of real-time imaging detection. Figure6 is image of enlarged workpiece. Comparing the results of real-time imaging detection and the results of film imaging detection, we can see the defect of $0.4 \mathrm{~mm}$ clearly, which meets the requirement of parts detection. The time of detection is about one-tenth of film detection. Time is saved and production proficiency is improved. Meanwhile, there is no need to take photos, so films and film potion are saved to reduce the costing of detection.

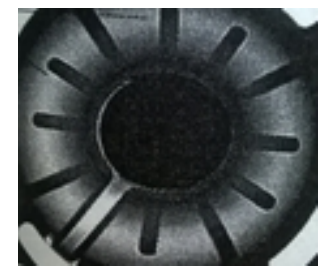

Figure5 Image of workpiece 


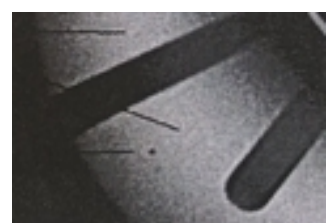

Figure6 Image of enlarged workpiece

We detect the experimental X-ray real-time imaging system according to national standards. Resolution ratio is $32 \mathrm{Lp} / \mathrm{cm}$, and sensitivity is $1.8 \%$.

The ratio of the defect measurement by theoretical analysis and the measurement of detected workpiece is $4 \sim 5 \%$, which meets the requirement of sensitivity detection. The detection result of system resolution $32 \mathrm{Lp} / \mathrm{cm}$ shows that the resolution of image intensifier doesn't make full use of its advantages. The detect of $0.2 \mathrm{~mm}$ can be detected based on the theoretical value of $58 \mathrm{Lp} / \mathrm{cm}$. With the improvement of computer image collection and processing system, the precision of detection can be perfected further.

\section{Conclusion}

The above technology research and detection experiment prove that X-ray real-time imaging detection technology is a practical way for high-precision detection on aluminum casting. Film imaging detection can be replaced completely, if the detection resolution is $32 \mathrm{Lp} / \mathrm{cm}$, and sensitivity is $1.8 \%$. Functions of static and dynamic transformation, electronic photoing, spot film, transformation of positive and negative film, measurement, video storage, video playback, image storage, image searching, image enlarging, image shrinking, conversion of false-color and gray level and image printing can be realized with computer image processing software. The technology and its equipment have been used by manufacturers because they can improve production efficiency, reduce the costing of production and offer convenient service on parts management to consumers.

\section{References}

[1] FuXinji, HanYan. Research on real-time intensifier technology of pipe welds XC-ray image sequence.J. Journal of Test and Measurement Technology. 15(2001)253-256.

[2] LiuDezhen. Modern Ray Testing Technology. Beijing: China Standards Press. 1999.

[3] QiangTianpeng. Ray Testing. Kunming: Yunnan Science and Technology Press, 2001.

[4] Xu Xiangxing. C/SiC Composites X-ray Nondestructive Testing.Northwestern Polytechnical University.2003

[5] Xu Li,Zhang Xinghong,Han Jiecai. Application of Radiographic Inspection Methods for Composite.NDT. 26(2004)450-456

[6]Jiang Xiaowei. Graphic Region Detection and Vehicle Detection System Based on X-ray. JiLin University.2014 